\title{
Correction: Sordid genealogies: a conjectural history of Cambridge Analytica's eugenic roots
}

\author{
Michael Wintroub
}

Correction to: Humanities and Social Sciences Communications https://doi.org/10.1057/s41599-020-0505-5, published online 17 July 2020.

The first two paragraphs in the section 'The ocean of computational politics' previously read:

The data collected, and subsequently "weaponized" by Cambridge Analytica, was sourced through the misappropriation of third party apps, such as "MyPersonality", developed by Michael Kosinski and David Stillwell, two psychologists working at Cambridge University's Psychometric Center. ${ }^{2}$ Posted on Facebook, these apps lured millions of users down the rabbit hole of clickbait personality tests grounded in the Five Factors of Personality theory, or OCEAN, an acronym for the personality attributes these tests are said to identify: Openness, Conscientiousness, Extraversion, Agreeableness, and Neuroticism. These traits, according to personality psychologists, provide the "objective" foundations for the study of human behavior. As one of the psychologist most closely associated with the Big Five, Paul Costa proclaimed: they "may be a human universal" (Quoted in Murphy Paul, 2004, p. 191). Objective or not, seen through the lens of digital data, they are said to be powerful predictive tools. Kosinski's team, for example, compared the results gleaned from these apps with all sorts of other online data: "likes" and "shares", and with information found in Facebook profiles and on-line postings. ${ }^{3}$ Cross-referenced and correlated, this data enabled researchers to construct algorithms that could infer personality traits from online activity alone, that is, without responses from on-line personality tests. Psychological profiles could thus be extrapolated by reference to online user behavior. This increased the data sample exponentially, allowing data scientists to track digital footprints back to "personality traits" that could then be hunted down and exploited in the "wild".

Cambridge Analytica's researchers claimed to have hi-jacked this "hack", thus allowing it to work with Facebook activity and map it onto the "Big Five" personality model. As their website advertised, using this data Cambridge Analytica could "accurately predict" with as few as 170 Facebook likes per Facebook user: ethnicity (95\%), gender (93\%), sexual orientation (88\%), politics (85\%), religion (82\%), relationship status (67\%), etc. With as many as 300 likes, they declared, they would "know you better than your spouse" (Carroll, 2017). Prior to the 2016 presidential election, Cambridge Analytica claimed to have acquired detailed personal data on 87 million Facebook users, while Alexander Nix, Cambridge Analytica's CEO, boasted that they had developed the means "to determine the personality of every single adult in the United States of America” (Kranish, 2016). Moreover, the model CA lifted from Kosinski and his group enabled Cambridge Analytica's researchers to extend both the reversibility and scalability of their stolen data. In other words, it allowed them to translate digital traces left in social media into the Five-Factor Personality Profile, and then to identify and search for specific profiles in the larger population-e.g., angry voters, resentful white men, undecided democrats, etc. (Matz et al., 2017).

A revision has been made to make clear that the authors do not suggest a connection between the data, algorithms, or expertise of Michal Kosinski and David Stillwell and their myPersonality App, and the work of Cambridge Analytica.

The revised text now reads:

In 2007, David Stillwell, a psychologist at Cambridge University, created a Facebook app called "MyPersonality” that allowed users to take a Big Five personality test measuring the OCEAN personality traits: Openness, Conscientiousness, Extraversion, Agreeableness, and Neuroticism. ${ }^{2}$ These traits, according to personality psychologists, provide the "objective" foundations for the study of human behavior. As one of the psychologist most closely associated with the Big Five, Paul Costa proclaimed: they "may be a human universal" (Quoted in Murphy Paul, 2004, p. 191). Objective or not, seen through the lens of digital data, they are said to be powerful predictive tools. The "MyPersonality" app took off with over 4 million users completing a personality test and about a third of them opting into sharing their Facebook profile data as well including their Facebook Likes. In 2013, a graduate student based at Cambridge University's Psychometrics Centre, Michal Kosinski, teamed up with Stillwell to publish research showing that Big Five personality and many other intimate traits could be predicted from Facebook Likes. Their algorithms could infer personality traits from online activity alone, that is, without responses from on-line personality tests. Psychological profiles could thus be extrapolated by reference to online user 
behavior. ${ }^{3}$ This increased the data sample exponentially, allowing data scientists to track digital footprints back to "personality traits" that could then be hunted down and exploited in the "wild". The research warned that algorithmic predictions could be "applied to large numbers of people without obtaining their individual consent and without them noticing" (Kosinski, 2013). As few as 170 Facebook likes per Facebook user could "accurately predict": ethnicity (95\%), gender (93\%), sexual orientation (88\%), politics (85\%), religion (82\%), relationship status (67\%), etc. With as many as 300 likes, they declared, they would "know you better than your spouse" (Carroll, 2017).

The year after their research was published, Cambridge Analytica tried hiring Kosinski and Stillwell but were rebuffed. Instead, CA teamed up with the psychologist Aleksandr Kogan. It has been reported that Kogan created his own personality testing Facebook app called "ThisIsYourDigitalLife" and then paid around 250,000 Americans a few dollars each to complete his personality test and share their Facebook profile data. Critically, users did not just share their own data but also shared the Facebook Likes of their friends, which was possible on Facebook at the time-this increased the sample size by 300 times. Armed with his own data, Kogan developed personality prediction algorithms and provided them to Cambridge Analytica. Prior to the 2016 presidential election, Cambridge Analytica claimed to have acquired detailed personal data on 87 million Facebook users, while Alexander Nix, Cambridge Analytica's CEO, boasted that they had developed the means "to determine the personality of every single adult in the United States of America" (Kranish, 2016). In other words, it allowed them to translate digital traces left in social media into the Five-Factor Personality Profile, and then to identify and search for specific profiles in the larger population-e.g., angry voters, resentful white men, undecided democrats, etc. (Matz et al., 2017).

Published online: 27 August 2020

Open Access This article is licensed under a Creative Commons Attribution 4.0 International License, which permits use, sharing, adaptation, distribution and reproduction in any medium or format, as long as you give appropriate credit to the original author(s) and the source, provide a link to the Creative Commons license, and indicate if changes were made. The images or other third party material in this article are included in the article's Creative Commons license, unless indicated otherwise in a credit line to the material. If material is not included in the article's Creative Commons license and your intended use is not permitted by statutory regulation or exceeds the permitted use, you will need to obtain permission directly from the copyright holder. To view a copy of this license, visit http://creativecommons.org/licenses/by/4.0/.
}

(C) The Author(s) 2020 